phosphatase being a chance finding. However when we reviewed the cases reported in the medical literature between $40-60 \%$ did actually have symptoms of failure to thrive or gastrointestinal disturbance. ${ }^{1-4}$ This therefore appears to be a common clinical association.

In all our cases radiology and other liver function tests were normal. Isoenzymes were measured in six cases and all but one showed either a mixture of liver and bone activity or bands between the two, typical of THI. There was a tendency for our cases to cluster during the autumn and winter months; this has been previously documented. ${ }^{4}$ Although the aetiology of THI remains unclear it may be the end result of different insults (infective or otherwise) in different children ${ }^{3}$; rotavirus was found in one of our cases and adenovirus in another. The importance of the diagnosis currently is its recognition, and in the avoidance of extensive investigations. Other diseases associated with such raised alkaline phosphatase would have clinical correlates such as deranged liver function tests, or abnormal wrist or hand radiographs. A family history of familial study would detect the rare familial type of raised alkaline phosphatase.

We suggest that the isolated finding of massively raised alkaline phosphatase in an infant is assumed to be THI unless clinical circumstances suggest otherwise. Isoenzyme analysis and other investigations of bone and liver need only be undertaken after eight weeks if the alkaline phosphatase has not begun to fall. Finally as between a half and three quarters of cases present with the related symptoms of diarrhoea, malabsorption or failure to thrive, we suggest that THI should no longer be considered as a pure biochemical phenomenon but rather a condition that does appear to have definite clinical associations.

E M BAILDAM M Z MUGHAI Department of Child Health, St Mary's Hospital, Manchester M13 OfH

M DALTON M A LEWIS
Royal Manchester Children's Hospital,
Pendlebury

Manchester M27 1HA

1 Kraut JR, Metrick M, Maxwell N, Kaplan M Isoenzyme studies in transient hyperphosphatasaemia of infancy. Ten new cases and a review of the literature. Am F Dis Child 1985; 139: $736-40$

2 Posen S, Lee C, Vines R, Kilham H, Latham S, Keefe JF. Transient hyperphosphatasaemia of infancy - an insufficiently recognised syninfancy - an insufficiently recog

3 Kruse K, Kurz N. Further evidence for infectious origin of isolated transient hyperphoorigin of isolated transient hyperphosphatasaemia. Eur $\mathcal{F}$ Padiatr 1989; 148: 453-4.
Crofton PM. What is the cause of benign transient hyperphosphatasaemia? A study of 35 cases. Clin Chem 1988; 34: 335-40.

\section{Dual marker one day pancreolauryl test}

EDITOR,-Dr Green and colleagues present interesting data relating to a novel 'tubeless' test of pancreatic exocrine function. ${ }^{1}$ However, their claim that this will greatly simplify the investigation of pancreatic exocrine dysfunction in childhood seems as difficult to swallow as $200 \mathrm{ml}$ of mannitol solution and $500 \mathrm{ml}$ of clear fluid! None of the controls were children and those patients with cystic fibrosis ranged up to 25 years of age. For various reasons some tests which perform well in adults turn out to be less useful in young children. ${ }^{2}$ As paediatricians, it is likely to be the infant with diarrhoea and failure to thrive whom we wish to investigate. As pancreatic insufficiency is relatively rare compared with other causes of this presentation, an investigation with high specificity is essential. As the authors have yet to produce any data on this crucial aspect of their test, may I recommend a simple, cheap, sensitive, specific, and repeatable alternative for which patients are generally only too happy to provide specimens - faecal chymotrypsin measurement. ${ }^{3}$ The dual marker pancreolauryl test is promising but needs further evaluation; I hope it has not been patented prematurely.

J W L PUNTIS
Neonatal Unit,
Clarendon Wing,
General Infirmary at Leeds,
Belmont Grove,
Leeds LS2 9NS

Green MR, Austin S, Weaver LT. Dual marke one day pancreolauryl test. Arch Dis Child 1993, 68: 649-52.

2 Sacher $M$, Kobas A, Shmerling DH. PABA screening test for exocrine pancreatic function in infants and children. Arch Dis Child 1978; 53 639-41.

3 Brown GA, Sule D, Williams J, Puntis JWL, Booth IW, McNeish AS. Faecal chymotrypsin a reliable index of exocrine pancreatic function. Arch Dis Child 1988; 63: 785-9.

\section{Epilepsy in children and the risk of drowning}

EDITOR,-Drs Kemp and Sibert raised the issue of death by drowning in children with epilepsy. ${ }^{1}$

We recently had a patient aged 10 years who had had epilepsy for four years and who was on treatment with sodium valproate. $\mathrm{He}$ had initially presented with frequent absences and these lasted only for a few seconds at a time. He had no other neurological problems. Before his death he had had no fits for the previous three weeks and had never had any generalised tonic-clonic seizures. $\mathrm{He}$ was found by his parents drowned in his bath having been in the bath for 5-10 minute period; he was resuscitated and was admitted to this hospital to the intensive care unit. On admission he was comatose, his pupils were fixed and dilated. He was treated by intermittent positive pressure ventilation, fluid restriction, and anticonvulsants. $\mathrm{He}$ developed further seizures the next morning, associated with hyperthermia and hypertension, and died 14 hours after the initial drowning episode. Postmortem examination showed presence of cerebral oedema and a few anoxic changes within his brain.

His history is a little similar to subject 1 in Kemp and Sibert's paper, that is, a child with normal intellect and no neurological signs who had had only a fairly minor form of epilepsy and no major motor problems. His death underlines the importance of supervision in the situation where drowning is a potential problem. All these cases support the view that all parents who have children, even if they have minor seizures, must either arrange for supervision of their children while they are in the bath or as suggested by Kemp and Sibert use a shower in an unlocked room.

$$
\begin{array}{r}
\text { MARK REID } \\
\text { Royal Belfast Hospital for Sick Children, } \\
180 \text { Falls Road, } \\
\text { Belfast BT12 } 6 B E
\end{array}
$$

1 Kemp AM, Sibert JR. Epilepsy in children and the risk of drowning. Arch Dis Child 1993; 68: 684-5.
Injury epidemiology: emerging statistics and strategy

EDITOR,-Recent articles in this journal are not only in tune with the World Health Organisation (WHO) message of the year 'handle life with care; prevent violence and negligence', but also have added significance to Third World readers. ${ }^{1-3}$ International trends in pedestrian injury mortality support the view that strategies directed towards upgrading the environment pay more dividends than educational awareness programmes. ${ }^{4} 5$ However such conclusions may unwittingly convey to developing countries that these strategies are mutually exclusive. For instance, in a symposium on injury prevention and social responsibility organised in this institute on WHO day, the delegates were equivocal in their emphasis on the environment and education. We think that these approaches are interdependent and reflect the dynamics of socioeconomic and political processes of a particular society. Improvements in the environment cannot come about without society demanding it, and this later phenomenon of peoples' assertion is unlikely to come from populations who lack awareness and education.

In the epidemiology of injury the conventional classification of children's mortality statistics into an age group 5 to 14 years fails to differentiate and delineate the prevention of risks peculiar to distinct lifestyles of children who are aged less than 9 and those who are older. Instead, for the epidemiology of injury children should be subdivided into 5-9 and 10-14 year age groups. This would encourage a better epidemiological understanding of age related problems and facilitate effective interventions in the hazardous host-agent-environment relationship. For example, children under the age of about 9 years do not have the necessary perceptual abilities to assess all the sensory inputs of the accident scenario such as traffic signals, etc, and hence they are unable to make a quick decision to avoid the accident. For this particular age group education of the parents and environmental changes play a major part compared with education aimed at modifying the behaviour of children. This latter strategy impinges upon the normal development of children by suppressing their natural instincts and curiosity for exploring their environment. On the other hand children older than 9 may benefit from educational programmes to boost the effects of environmental measures.

L R MURMU Department of Surgery SHASHI KANT Department of Community Medicine, All India Institute of Medical Sciences, New Delhi 110029 ,

1 Roberts IG. International trends in pedestrian injury mortality. Arch Dis Child 1993; 68: 190-2.

2 Gofin R, Lison M, Morag C. Injuries in primary care practices. Arch Dis Child 1993; 68: 223-6.

Rogers M. Cycle helmets. Arch Dis Child 1993; 68: 237-9.

4 Sibert JR. Accidents to children: the doctor's role education or environmental change? Arch Dis Child 1991; 66: 890-3.

5 Grayson GB. The identification of training objectives: what shall we tell the children? Accid Anal Prev 1981; 13: 169-73.

\section{Pressure reduction of intussusception}

EDITOR,-In this January's issue of the journal you published a paper from this centre 
concerning pressure reduction of intussusception. ${ }^{1}$ In this paper the success rate for reduction was quoted at $76 \%$ when barium was used and $64 \%$ when air was used, giving an overall success rate of $66 \%$.

I would like to point out that the cases reported by den Hollander and Burge spanned the time that the Wessex Department of Paediatric Radiology was converting from the use of barium to air as a reduction medium, and we were relatively inexperienced in the use of air. With increasing experience our success rate has improved considerably, and a recent audit of 58 intussusception reductions performed in our department showed that the reduction rate with air had increased to $86 \%$. This compares well with figures published from all other centres as far as I am aware.

J FAIRHURST

Wessex Department of Paediatric Radiology, Southampton General Hospital,

Tremona Road,

Southampton SO9 $4 X Y$

1 Den Hollander D, Burge DM. Exclusion criteria and outcome in pressure reduction of intussusception. Arch Dis Child 1993; 68: 79-81.

\section{A career in paediatrics? A survey of paediatric senior house officers in England and Wales}

EDITOR,-Barker and Buss documented that $32 \%$ of senior house officers (SHOs) in their study reported below average levels of in-post training. ${ }^{1}$

The Department of Health and the Northern and East Anglian Postgraduate Deans have funded a pilot study in Cambridge, Norwich, Newcastle, and Sunderland to improve paediatric training for SHOs. The strength of the scheme is that it is integrated with the SHO's working day. The scheme which started on 1 March 1993 is called 'Service-Based Learning'2 and is divided into four parts. The first is 'practice based learning' and involves spending about 15 minutes discussing with the SHOs a topic of interest that arose on a ward round or an outpatient clinic. A learning plan is then devised, which may involve reading a departmental protocol or visiting a specialist clinic, or undertaking any other activity to complete the study of that topic. The second component comprises 'brief learning materials' (BLMs) and is due to start shortly. There are over 200 BLMs which cover the paediatric curriculum and which were written by 75 registrars and consultants for this study. They are symptom orientated, often have a question and answe format, and take about 15 minutes to read. The BLMs are organised in topic groups, each of which has an associated self assessment. Model answers are held by seniors. The learning materials are located on the wards and are for reading by the SHOs during gaps in the day and while on call. The third component covers departmental meetings. These must be interactive with discussion, rathe than formal 'spoon feeding' type lectures. The fourth constitutes a period of study leave of up to one week, which must be arranged at the beginning of the post.

The SHOs have a booklet in which they record what they have learnt and in which the registrar and consultant sign up the learning periods. They also meet regularly with the clinical tutor to ensure they are progressing satisfactorily. The aim is to accumulate 100 or more learning credits, each credit being equivalent to one hour's learning. Upon achieving 100 credits the SHO is awarded a service based learning certificate which should be mentioned in their curriculum vitae.

If this pilot study proves successful the scheme will be introduced to other regions. Together with the changes that will accompany the Calman report we hope that this scheme will improve the paediatric training of SHOs and make their jobs more interesting.

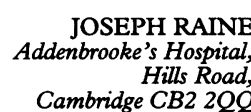
JANET GRANT
foint Centre for Education in Medicine Foint Centre for Education in Medicine,
(British Postgraduate Medical Federation/Open University) 33 Millman Street,
London WC1N $3 E \mathcal{F}$

1 Barker DP, Buss PW. A career in paediatrics? A survey of paediatric senior house officers in
England and Wales. Arch Dis Child 1993; 68: 752-3.

2 Grant J, Marsden P. Training senior house officers by service-based learning. BPMF, London: The Joint Centre for Education in Medicine, 1992.

The Australian NHMRC Twin Registry: a resource for paediatric research

EDITOR,-The Australian NHMRC Twin Registry contains over 4000 pairs of twins under 10 years old, and a further 3000 up to the age of 18 years. The parents of these twins have volunteered to consider requests from researchers for studies in bona fide projects approved by the registry. This represents a major resource for studies in paediatric and adolescent epidemiology.

There are numerous and ingenious ways in which twins can be used to address scientific and medical questions. Some examples include examining genetic and environmental causes of variation, co-twin control studies based on disease discordant or exposure discordant pairs, longitudinal studies, and studies of gene-environment interaction. ${ }^{1}$

Although there have been over 70 projects conducted over the last 12 years on the 17000 adult pairs on the registry, to date there have been few studies making use of the younger pairs. Baseline information has been computerised on about $50 \%$ of these pairs.

I would like to bring this to the attention of international paediatric researchers. Applications to utilise the registry can be made from researchers throughout the world. There may be some charge, depending on costs and the level of work required by local staff. All applications are reviewed by the executive committee of the registry, and advice on study design and practicalities is available. For more information, please write to me at the address below.

JOHN L HOPPER Australian NHMRC Twin Registry, 151 Barry Street, Carlton, Victoria 3053, Australia

1 Clifford CA, Hopper JL. The Australian NHMRC Twin Registry. A resource for the Australian scientific community. Med $\mathcal{F}$ Aust 1986; 145: 63-5. 